\title{
A comparative study of the molecular evolution of signalling pathway members across olfactory, gustatory and photosensory modalities
}

\author{
CHAO HE ${ }^{1,2}$, DAVID A. FITZPATRICK ${ }^{3}$ and DAMIEN M. O'HALLORAN ${ }^{1,2 *}$ \\ ${ }^{1}$ Department of Biological Sciences, George Washington University, 333 Lisner Hall, 2023 G St. NW, Washington, \\ DC 20052, USA \\ ${ }^{2}$ Institute for Neuroscience, George Washington University, 636 Ross Hall, 2300 I St. NW, Washington DC 20037, USA \\ ${ }^{3}$ Department of Biology, Genome Evolution Laboratory, National University of Ireland Maynooth, Maynooth, \\ Co. Kildare, Ireland
}

[He C., Fitzpatrick D. A. and O'halloran D. M. 2013 A comparative study of the molecular evolution of signalling pathway members across olfactory, gustatory and photosensory modalities. J. Genet. 92, 327-334]

\section{Introduction}

All sensory modalities serve a similar objective, which is to decode input by making predictions in time and space about an animal's surroundings. The evolution of sensory modalities is driven by the need to shape effective behavioural outputs, and in turn increase survival. Throughout evolution, sensory systems have undergone a great deal of specialization; and even though some modalities are derived from unique origins within different phyla, they still exhibit many common design features (Strausfeld and Hildebrand 1999; Eisthen 2002; Jacobs et al. 2007). We now have detailed mechanistic data on how sensory systems operate within specific animals (Buck and Axel 1991; Chalasani et al. 2007; Sato et al. 2008; Wicher et al. 2008), however it is still not clear how sensory signalling pathways evolve at the molecular level, and whether these evolutionary mechanisms are shared between diverse taxa. Here we set out to investigate the molecular evolution of signalling pathway members across olfactory, gustatory, and photosensory modalities from very divergent phyla in an attempt to develop a model of molecular evolution for sensory systems. From our pairwise intraphylum analysis we found that sensory signalling pathways unusually undergo high levels of functional constraint that are higher than genomewide global levels of constraint, and this purifying selection is common within the very divergent taxa we examined. We also find that gene duplication events represent a conserved but heterogeneous driver of evolution within sensory signalling pathways. Taken together, we propose a 'sessile' mechanism of sensory signalling pathway evolution, which on one side

*For correspondence. E-mail: damienoh@gwu.edu. facilitates bursts of gene duplication and relaxed selection and on the other side it is unusually anchored by high levels of selective constraint that preserves core sensory function.

\section{Materials and methods}

\section{Sequences}

Sequences were sourced from the Kyoto Encyclopedia of Genes and Genomes (KEGG, http://www.genome.jp/kegg/) pathway database by following the data oriented entry point pathway. Pathways that were not represented at KEGG were sourced from Flybase ver. FB2012.05 (http://flybase.org/), and Wormbase ver. WS233 (http://www.wormbase.org). Only genes that had clear intraphylum orthologues from the database InParanoid7 (http://inparanoid.sbc.su.se) or OrthoDB (http://cegg.unige.ch/orthodb6) were selected and only reference (or longest) splice forms were included. Paralogues were sourced using maximum stringency settings from the KEGG pathway sequence similarity database (SSDB), which contains the information about amino acid sequence similarities among all protein-coding genes in completed genomes using Smith-Waterman similarity scoring and bidirectional best hits.

\section{Phylogenetic analysis}

Phylogenetic relationships were inferred by reconstructing phylogenies via maximum likelihood using PhyML (Guindon and Gascuel 2003). Orthologues were aligned using the multiple sequence alignment software MUSCLE ver. 3.8.31 (Edgar 2004), and gaps were systematically stripped from all sequences after alignment.

Keywords. olfaction; gustation; photo-excitation; comparative evolution; divergence; molecular diversity; gene duplication. 


\section{Analysis of molecular data}

Synonymous $\left(d_{\mathrm{S}}\right)$ and nonsynonymous $\left(d_{\mathrm{N}}\right)$ substitution rates for orthologues were estimated using the methods of Yang and Nielsen (2000) as implemented in yn00 in the PAML suite (Yang 1997; Yang and Nielsen 2000) using an intraphylum pairwise approach. To compare the frequency of substitutions at silent sites to that of nonsilent sites within each pathway to the frequency of substitutions at silent sites to that of nonsilent sites across the genomes of the taxa under examination, randomization testing was determined. Randomization analysis was performed by calculating the average $d_{\mathrm{N}} / d_{\mathrm{S}}$ value for 50,000 randomly assembled groups of orthologous genes. Random networks were equal in size to the average size of our pathways and sampling permitted replacement. Only 1:1 protein coding orthologues that were represented by $100 \%$ bootstrap support at InParanoid 7 were included. Measures of nucleotide diversity $(\pi)$ were performed using DnaSP ver. 5 (Librado and Rozas 2009) from multiple sequence alignments generated using the software MUSCLE ver. 3.8.31 (Edgar 2004). The average paranome size within each phylum was computed by firstly performing a blast-all-against-all search within each genome and then generating groups using the Markov clustering algorithm (MCL), http://micans.org/mcl/index.html.

\section{Statistics}

All averaged data are expressed as mean \pm SEM. Statistical analysis was performed with Student's $t$-test and $P<0.05$ was considered statistically significant. Gene duplication correlations were measured using the nonparametric Spearman's rank correlation coefficient $\left(r_{s}\right)$ test, and also using the Pearson product-moment correlation coefficient $(r)$ using $\mathrm{R}$ project: http://www.r-project.org/.

\section{Results}

To examine the rate of divergence across olfactory, gustatory, and photosensory modalities within each of three phyla (Nematoda, Arthropoda and Chordata), we downloaded signalling pathway genes from KEGG. Pathways that were not represented at KEGG were sourced from flybase and wormbase databases. From this we compiled a list of genes in each sensory modality from the nematodes Caenorhabditis elegans and C. briggsae; from the insects Drosophila melanogaster and Anopheles gambiae; and from the mammalian species, human and mouse. Next we screened for those genes that are clear intraphylum orthologues between $C$. elegans and $C$. briggsae, or between D. melanogaster and A. gambiae, or between human and mouse (table 1). We examined the divergence rate between each pairing by comparing substitutions at silent sites to that of nonsilent sites for each intraphylum orthologous pairing. By comparing the average rate of divergence for all paralogues from each sensory modality we found that in each modality the divergence rate was not significantly different (figure 1, A$\mathrm{B} ; P=0.6$ for olfaction $d_{\mathrm{N}} / d_{\mathrm{S}}$ versus gustation; $P=0.5$ for olfaction $d_{\mathrm{N}} / d_{\mathrm{S}}$ versus phototransduction $d_{\mathrm{N}} / d_{\mathrm{S}} ; P=$ 0.62 for gustation $d_{\mathrm{N}} / d_{\mathrm{S}}$ versus phototransduction $d_{\mathrm{N}} / d_{\mathrm{S}}$; $P=0.07$ for olfaction $d_{\mathrm{N}}$ versus gustation $d_{\mathrm{N}} ; P=0.3$ for olfaction $d_{\mathrm{N}}$ versus phototransduction $d_{\mathrm{N}} ; P=0.28$ for gustation $d_{\mathrm{N}}$ versus phototransduction $d_{\mathrm{N}}$ ). We also determined the average extent of molecular diversity $(\pi)$ for each orthologue pairing, and found that in case of diversity there was no significant difference between modalities (figure $1 \mathrm{C}$; $P=0.1$ for olfaction versus gustation; $P=0.3$ for olfaction versus phototransduction; $P=0.7$ for gustation versus phototransduction).

Overall, we found that each modality is undergoing purifying selection (figure 1A). To place this rate of selection in a context of global divergence within each phylum we examined genomewide rates of divergence for: $C$. elegans versus C. briggsae; D. melanogaster versus A. gambiae; and for human versus mouse. We then generated randomized data sets $(50,000$ in total) within each phylum and examined the frequency of mean $d_{\mathrm{N}} / d_{\mathrm{S}}$ values in each case (figure $1, \mathrm{D}-\mathrm{F}$ ). From this analysis we found an average $d_{\mathrm{N}} / d_{\mathrm{S}}$ value $=0.14$ for human versus mouse, which is over 1.5 times higher than the average $d_{\mathrm{N}} / d_{\mathrm{S}}$ level within the olfactory signalling pathway (blue arrow), and over 2.5 times higher than the average $d_{\mathrm{N}} / d_{\mathrm{S}}$ level within the gustatory pathway (red arrow), or phototransduction pathway (green arrow) in mammals. In case of Drosophila versus Anopheles, we found an average $d_{\mathrm{N}} / d_{\mathrm{S}}$ across the genome of 0.11 ; this value is over five times higher than the average $d_{\mathrm{N}} / d_{\mathrm{S}}$ value for the olfactory signalling pathway (blue arrow), and almost an order of magnitude higher $(9.5 \times)$ than the average for the gustatory signalling pathway (red arrow), and almost 20 times higher (18.4) than the average value for the phototransduction cascade (green arrow) between flies and mosquitoes. In case of Caenorhabditis, we found an average $d_{\mathrm{N}} / d_{\mathrm{S}}$ value of 0.08 , which is between two to three times higher than the average constraint that is observed for the olfactory (blue arrow), gustatory (red arrow), or phototransduction (green arrow) cascades between $C$. elegans and $C$. briggsae. Our randomization data are consistent with previous reports on global $d_{\mathrm{N}} / d_{\mathrm{S}}$ range (Stein et al. 2003; Wang et al. 2007), and taken together with our data on sensory signalling pathways, this suggests that the overall conserved sensory signalling cascade members are under more functional constraint than the average level of global constraint across the genome in each case. Interestingly, we observed most constraint for the phylum Arthropoda, with the highest level of constraint detected for the phototransduction cascade, followed next by taste signalling, and then olfaction.

Next we examined the extent of gene duplication for each modality within each phylum by comparing the number of paralogues for each member of each signalling pathway. From this analysis we found significant gene duplications in each phylum and for each modality. Moreover, we revealed that in each phylum the extent of gene duplication was 
Table 1. List of genes, accession numbers, and the encoded proteins of all loci examined.

\begin{tabular}{|c|c|c|c|c|}
\hline Gene & ${ }^{7} \mathrm{KEGG}$ id & Ortholog & KEGG id & Protein \\
\hline \multicolumn{5}{|l|}{ Phylum chordata } \\
\hline${ }^{1}$ H. sap Arrestin & 409 & ${ }^{2} M . m u s$ Arrestin & 216869 & Beta arrestin-2 \\
\hline H. sap PKA & 5613 & M. mus PKA & 19108 & Protein kinase A \\
\hline H. sap $\mathrm{PKG}$ & 5593 & M. mus $\mathrm{PKG}$ & 19092 & Protein kinase $\mathrm{G}$ \\
\hline H. sap GCAP & 2978 & M. mus GCAP & 14913 & Guanylate cyclase activator $1 \mathrm{C}$ \\
\hline H. sap G-olf & 2774 & M. mus G-olf & 14680 & G protein $\mathrm{G}($ olf $)$ subunit alpha \\
\hline H. sap CaMKII & 817 & M. mus CaMKII & 108058 & $\mathrm{Ca}^{2+} / \mathrm{CaM}$-dependent protein kinase II $\delta$ \\
\hline H. sap $\mathrm{CaM}$ & 801 & M. mus CaM & 12313 & Calmodulin \\
\hline H. sap $\mathrm{CNG}$ & 54714 & M. mus $\mathrm{CNG}$ & 30952 & Cyclic nucleotide gated channel beta 3 \\
\hline H. sap AC & 109 & M. mus AC & 104111 & Adenylate cyclase \\
\hline H. sap $\mathrm{R}$ & 81466 & M. mus $\mathrm{R}$ & 258937 & Olfactory receptor \\
\hline H. sap $\mathrm{R} 1$ & 10798 & M. mus $\mathrm{R} 1$ & 258640 & Olfactory receptor \\
\hline H. sap $\mathrm{R} 2$ & 119687 & M. mus R2 & 258248 & Olfactory receptor \\
\hline H. $\operatorname{sap} \mathrm{CLC} \alpha$ & 1179 & M. mus CLC $\alpha$ & 23844 & Chloride channel calcium activated \\
\hline H. sap Phosducin & 5132 & M. mus Phosducin & 20028 & Phosducin \\
\hline H. sap Gustducin & 346562 & M. mus Gustducin & 242851 & G-protein $\mathrm{G}(\mathrm{t})$ subunit alpha \\
\hline H. sap GNB1 & 2782 & M. mus GNB1 & 14688 & $\mathrm{G}$ protein beta subunit \\
\hline H. sap $\mathrm{T} 1 \mathrm{R}$ & 83756 & M. mus T1R & 83771 & Taste receptor \\
\hline H. sap PDE & 5136 & M. mus PDE & 18573 & Calmodulin-dependent phosphodiesterase \\
\hline H. sap PLC $\beta$ & 5330 & M. mus PLC $\beta$ & 18796 & Phospholipase C beta \\
\hline H. sap $\mathrm{CaCN}$ & 773 & M. mus $\mathrm{CaCN}$ & 12286 & Voltage-dependent calcium channel P/Q type \\
\hline H. sap $\mathrm{KCN}$ & 3745 & M. mus $\mathrm{KCN}$ & 16500 & Potassium voltage-gated channel \\
\hline H. sap PKA & 5566 & M. mus PKA & 18747 & Protein kinase A (olfactory) \\
\hline H. sap $\mathrm{AC}$ & 196883 & M. mus AC & 104110 & Adenylate cyclase \\
\hline H. sap $\mathrm{ENaC}$ & 6337 & M. mus $\mathrm{ENaC}$ & 20276 & Non voltage-gated sodium channel 1 alpha \\
\hline H. sap Rec & 5957 & M. mus Rec & 19674 & Recoverin \\
\hline H. sap $\mathrm{Rh}$ & 6010 & M. mus $\mathrm{Rh}$ & 212541 & Rhodopsin \\
\hline H. sap Arr & 408 & M. mus Arr & 109689 & Beta arrestin-1 \\
\hline H. sap $\mathrm{Gt}$ & 2779 & M. mus Gt & 14685 & G-protein subunit alpha \\
\hline H. sap RGS9 & 8787 & M. mus RGS9 & 19739 & Regulator of $G$ protein signaling \\
\hline H. sap PDE & 5145 & M. mus PDE & 225600 & cGMP phosphodiesterase \\
\hline H. sap $\mathrm{GC}$ & 2986 & M. mus GC & 245650 & Guanylate cyclase $2 \mathrm{f}$ \\
\hline H. sap NCKX & 9187 & M. mus NCKX & 214111 & Sodium/potassium/calcium exchanger \\
\hline H. sap $\mathrm{CNG}$ & 1259 & M. mus $\mathrm{CNG}$ & 12788 & Cyclic nucleotide gated channel alpha 1 \\
\hline \multicolumn{5}{|l|}{ Phylum arthropoda } \\
\hline${ }^{3}$ D. me Ir8a-PA & Dmel CG32704 & ${ }^{4}$ A. $g a \operatorname{Ir} 8 \mathrm{a}$ & AgaP AGAP010411 & Ionotropic glutamate receptor \\
\hline D. me Galpha & Dmel_CG17759 & A. ga Galpha & AgaP_AGAP005079 & $\mathrm{G}$ protein alpha subunit \\
\hline D. me eag & Dmel CG10952 & A. ga eag & AgaP AGAP002719 & Voltage gated cation channel \\
\hline D. me geko & Dmel_CG13695 & A. ga geko & AgaP_AGAP005168 & Novel olfactory Gene \\
\hline D. me orco & Dmel CG10609 & A. ga orco & AgaP AGAP002560 & Odorant receptor co-receptor \\
\hline D. $m e$ dunce & Dmel_CG32498 & A. ga dunce & AgaP_AGAP000236 & cAMP phosphodiesterase \\
\hline D. $m e$ gcy $89 \mathrm{da}$ & Dmel_CG14885 & A. ga gcy $89 \mathrm{da}$ & AgaP_AGAP004564 & Atypical soluble guanylyl cyclase \\
\hline D. me gr5a & Dmel CG15779 & A. ga gr5a & AgaP AGAP003253 & Sweet taste receptor \\
\hline D. me gr66a & Dmel_CG7189 & A. ga gr66a & AgaP_AGAP002275 & Gustatory receptor \\
\hline D. $m e$ goa $47 \mathrm{a}$ & Dmel_CG2204 & A. ga goa $47 \mathrm{a}$ & AgaP_AGAP005773 & $\mathrm{G}$ protein alpha subunit \\
\hline D. me TRPA 1 & Dmel_CG5751 & A. ga TRPA 1 & AgaP_AGAP004863 & Transient receptor potential (TRP) A1 \\
\hline D. me AC78c & Dmel_CG10564 & A. ga AC78c & AgaP_AGAP002262 & Adenylyl cyclase \\
\hline D. $m e \mathrm{Gq}$ & Dmel_CG17759 & A. $g a \mathrm{Gq}$ & AgaP_AGAP005079 & G protein alpha subunit \\
\hline D. me PLC $\beta$ & Dmel_CG3620 & A. ga $\mathrm{PLC} \beta$ & AgaP AGAP001936 & Phosphatidylinositol phospholipase C \\
\hline D. $m e$ PKC & Dmel_CG6518 & A. ga $\mathrm{PKC}$ & AgaP_AGAP012252 & Protein kinase $\mathrm{C}$ \\
\hline D. $m e$ TRP & Dmel_CG5996 & A. ga TRP & AgaP_AGAP008435 & TRP cation channel family $\mathrm{C}$ \\
\hline D. me TRPL & Dmel_CG18345 & A. ga TRPL & AgaP_AGAP010630 & TRP-like cation channel \\
\hline D. me CaM & Dmel_CG8472 & A. ga CaM & AgaP AGAP010957 & Calmodulin \\
\hline D. $m e$ Arr-2 & Dmel_CG5962 & A. ga Arr-2 & AgaP_AGAP006263 & Phototransduction arrestin \\
\hline D. me INAD & Dmel_CG3504 & A. $g a$ INAD & AgaP_AGAP002145 & Inactivation-no-after-potential D protein \\
\hline D. $m e$ NINAC & Dmel CG5125 & A. ga NINAC & AgaP AGAP009730 & Neither inactivation nor after potential $\mathrm{C}$ \\
\hline D. me IP3R & Dmel_CG1063 & A. ga IP3R & AgaP_AGAP006475 & Inositol 1,4,5-triphosphate receptor \\
\hline
\end{tabular}


Table 1. (contd.)

\begin{tabular}{|c|c|c|c|c|}
\hline Gene & ${ }^{7} \mathrm{KEGG}$ id & Ortholog & KEGG id & Protein \\
\hline \multicolumn{5}{|c|}{ Phylum nematoda } \\
\hline${ }^{5}$ C. el odr- 10 & CELE C53B7.5 & ${ }^{6} C . b r$ odr- 10 & CBG10912 & De-orphaned olfactory receptor \\
\hline C. el str-2 & CELE_C $50 \mathrm{C} 10.7$ & C. $b r$ str-2 & CBG19383 & Orphan olfactory receptor \\
\hline C. el str-1 & CELE_C42D4.5 & C. $b r$ str- 1 & CBG05794 & Orphan olfactory receptor \\
\hline C. el arr-1 & CELE_F53H8.2 & C. $b r$ arr- 1 & CBG08085 & Arrestin \\
\hline C. el rgs-3 & CELE_C $29 \mathrm{H} 12.3$ & C. $b r$ rgs -3 & CBG02574 & Regulator of G protein signaling \\
\hline C. el grk-2 & CELE W02B3.2 & C. $b r$ grk-2 & CBG15249 & G-protein receptor kinase \\
\hline C. el odr-3 & CELE_C34D1.3 & C. $b r$ odr-3 & CBG09409 & G protein alpha subunit \\
\hline C. el egl-4 & CELE_F55A8.2 & C. $b r$ egl-4 & CBG08401 & Protein kinase $\mathrm{G}$ \\
\hline C. el tax-2 & CELE_F36F2.5 & C. $b r$ tax -2 & CBG12422 & CNG channel beta subunit \\
\hline C. el tax-4 & CELE_ZC84.2 & C. $b r$ tax -4 & CBG06949 & CNG channel alpha subunit \\
\hline C. el odr-1 & CELE_R01E6.1 & C. $b r$ odr- 1 & CBG07425 & Receptor guanylyl cyclase \\
\hline C. el daf-11 & CELE-B0240.3 & C. $b r$ daf -11 & CBG23280 & Receptor guanylyl cyclase \\
\hline C. el gpa-3 & CELE_E02C12.5 & C. $b r$ gpa-3 & CBG19263 & $\mathrm{G}$ protein alpha subunit \\
\hline C. el gpc-1 & CELE_K02A4.2 & C. $b r$ gpc-1 & CBG00049 & G protein gamma subunit \\
\hline C. el gcy-7 & CELE F52E1.4 & C. br gcy-7 & CBG11271 & Receptor guanylyl cyclase \\
\hline C. el gcy-5 & CELE_ZK970.6 & C. $b r$ gcy-5 & CBG00850 & Receptor guanylyl cyclase \\
\hline C. el osm-9 & CELE B0212.5 & C. $b r$ osm-9 & CBG15022 & TRP cation channel \\
\hline C. el lite-1 & CELE_C14F11.3 & C. $b r$ lite- 1 & CBG05007 & Light activated 8-TM protein \\
\hline C. el goa-1 & CELE_C26C6.2 & C. $b r$ goa- 1 & CBG24698 & G protein alpha subunit \\
\hline C. el pde-1 & CELE_T04D3.3 & C. $b r$ pde- 1 & CBG20362 & cGMP phosphodiesterase \\
\hline C. el pde-2 & CELE_R08D7.6 & C. $b r$ pde- 2 & CBG06847 & cGMP phosphodiesterase \\
\hline C. el pde-5 & CELE_C32E12.2 & C. $b r$ pde- 5 & CBG12836 & cGMP phosphodiesterase \\
\hline
\end{tabular}

${ }^{1}$ H. sap refers to Homo sapiens

${ }^{2}$ M. mus refers to Mus musculus

${ }^{3} D$. me refers to Drosophila melanogaster

${ }^{4}$ A. ga refers to Anopheles gambiae

${ }^{5}$ C. el refers to Caenorhabditis elegans

${ }^{6} \mathrm{C}$. br refers to Caenorhabditis briggsae

${ }^{7}$ KEGG refers to the 'Kyoto Encyclopedia of Genes and Genomes' (http://www.genome.jp/kegg/).

highest for the olfactory modality, and this was especially true for nematodes and mammals where the number of duplicates were significantly higher for the olfactory signalling pathway when compared with the phototransduction cascade (figure $2 \mathrm{~A}, P=0.02$ for $C$. elegans olfactory versus $C$. elegans phototransduction; $P=0.023$ for $C$. briggsae olfactory versus $C$. briggsae phototransduction; $P=0.02$ for mouse olfactory versus mouse phototransduction; $P=0.03$ for human olfactory versus human phototransduction). By averaging the number of paralogues across all phyla and partitioning by sensory modality we observe almost seven times as many duplicates within the olfactory circuit than the phototransduction circuit, and over four times as many paralogues for the olfactory circuit than the gustatory circuit (figure 2B), revealing a modality specific expansion of the olfactory circuit. To develop an understanding of global levels of gene duplication within each phylum, we generated the paranome (number of proteins with one or more paralogues) for C. elegans, D. melanogaster, and Mus musculus using the MCL clustering algorithm (http://micans.org/mcl/) and found the median gene family to be between two and three members in size. The difference in global gene family size compared with gene families within sensory systems (figure 2B) clearly underscores the extent to which gene duplication impacts the sensory systems and highlights the striking spike in gene duplication observed within the olfactory system, and to a lesser extent the gustatory and photosensory systems (figure 2B). Following this we examined the level of concordance for gene duplication events for each phylum and within each sensory modality. For each sensory modality we observed robust correlations between the number of paralogues within each modality (figure 2, C-E: i, nematode; ii, insect; iii, mammal-significance at $P<0.001$ using Spearman rank-order correlation coefficient, $r_{\mathrm{s}}$, and coefficient of determination, $r^{2}$ ). Within arthropods overall we observed more narrow expansions (figure 2A). We also observed fewer clear orthologues within the sensory systems of $D$. melanogaster and $A$. gambiae (figure 2, Cii-Eii), likely reflecting in part their more ancient divergence estimate of 250 mya (Gaunt and Miles 2002). As mentioned above, we also observed most constraint within the arthropod sensory pathway, which also likely reflects this greater evolutionary distance as we only included orthologues supported by $100 \%$ bootstrap support. Divergence estimates for human with mouse and $C$. elegans with $C$. briggsae are between 80-95 mya (Nei et al. 2001; Stein et al. 2003). We also observed a significantly reduced olfactory receptor repertoire in humans when compared to mouse (figure 2 Ciii) which is 
A

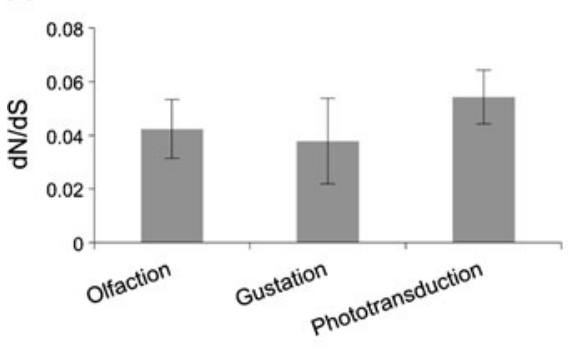

D

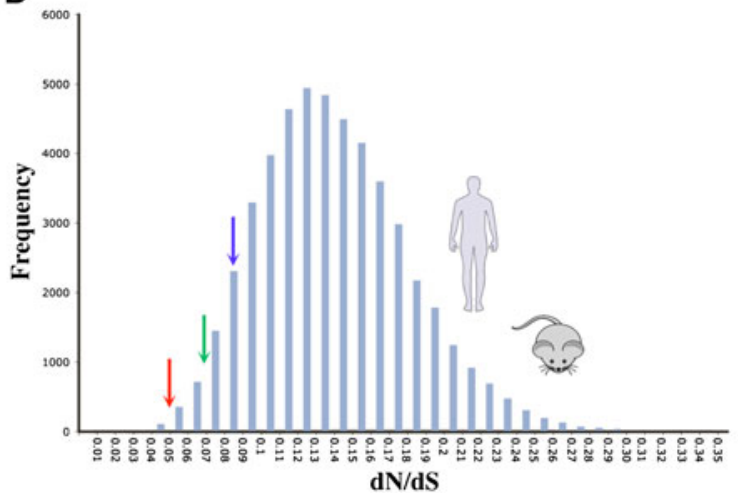

$\mathbf{F}$

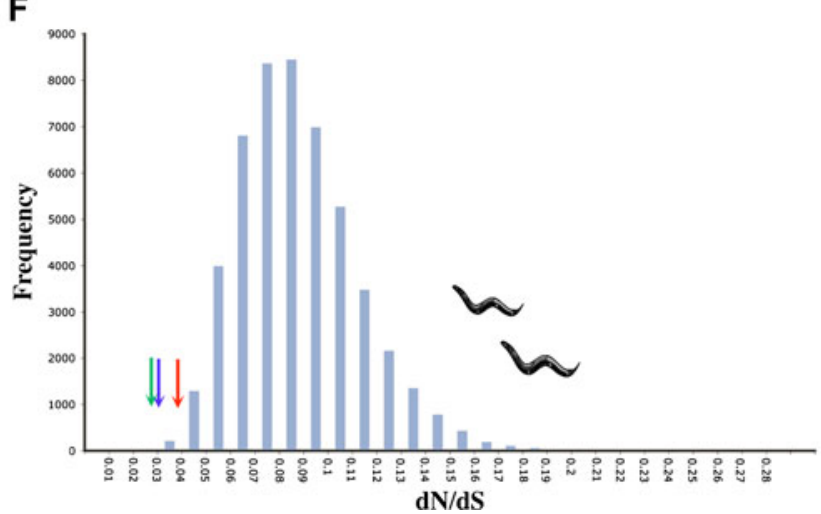

B
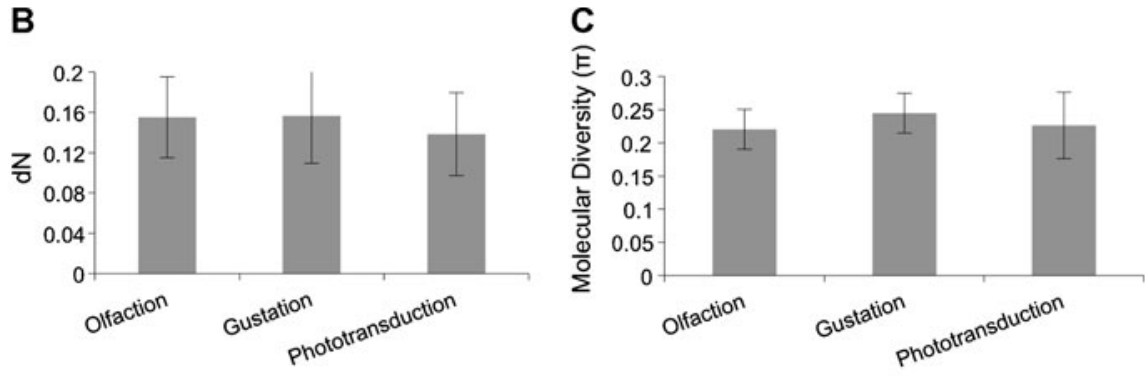

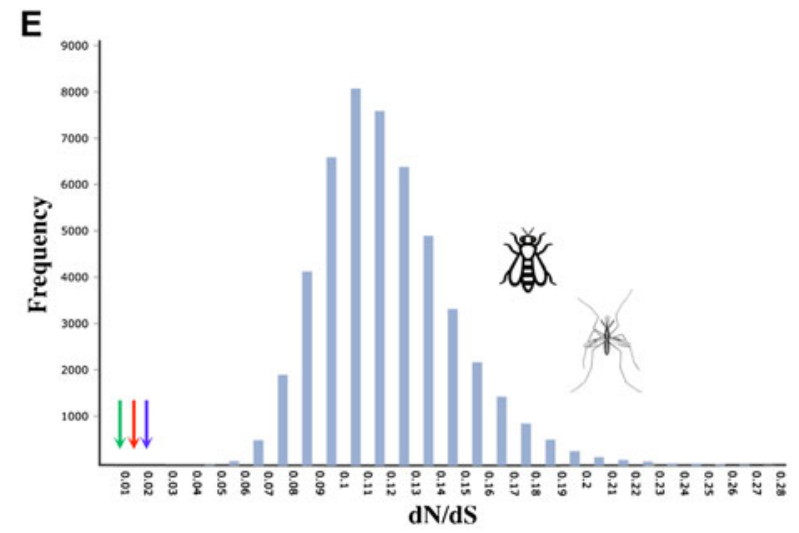

Figure 1. Average rates of divergence and diversity across all phyla partitioned by sensory modality. (A) Plot of average divergence values for each sensory modality from nematodes, insects and mammals. (B) Plot of average number of nonsynonymous substitutions per nonsynonymous site for each sensory system from nematodes, insects and mammals. (C) Plot of average molecular diversity for each sensory system across nematodes, insects, and mammals. Error bars represent the standard error of the mean. (D-F) Frequency distribution of average $d_{\mathrm{N}} / d_{\mathrm{S}}$ values from 50,000 randomization sets (blue bars) comprising orthologous groupings from mammals (D), insects (E) and nematodes (F). The $x$-axis in each case represents binned divergence categories plotted against the frequency on the $y$-axis. The blue arrows indicate the average $d_{\mathrm{N}} / d_{\mathrm{S}}$ value of the olfactory sensory system in each case, the red arrows indicates the average $d_{\mathrm{N}} / d_{\mathrm{S}}$ value of the gustatory network in each case, and the green arrows indicates the average $d_{\mathrm{N}} / d_{\mathrm{S}}$ value of the photoexcitation cascade in each case.

consistent with previous descriptions of the human olfactory receptor family (Young et al. 2002).

\section{Discussion}

\section{Purifying selection and sensory systems}

From our results we found that the overall function of conserved signalling pathway members within the olfactory, gustatory, and photoexcitation systems is preserved through intense constraint at the molecular level which is higher than the level of global constraint across the genome in each case.
By examining gene duplication events within each sensory system, we observed significant expansions in each case, with the olfactory system exhibiting the greatest number of duplicates. Taken together our data suggests that sensory systems appear to evolve largely through gene duplication events, which provides a pool of new genes that likely undergo such a relaxed level of selective constraint that identifying clear orthologues is very difficult and not likely to be detected through our analysis. However, in order to balance this strategy of large gene duplication events, the core signalling pathway members endure unusually strong purifying selection to preserve their critical function. 
A

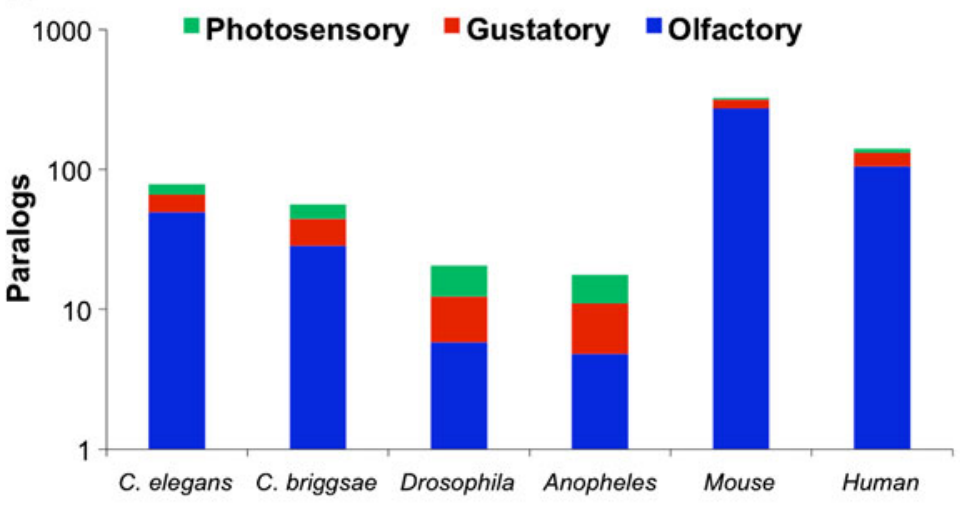

C

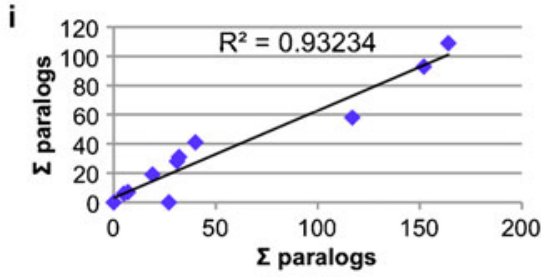

ii
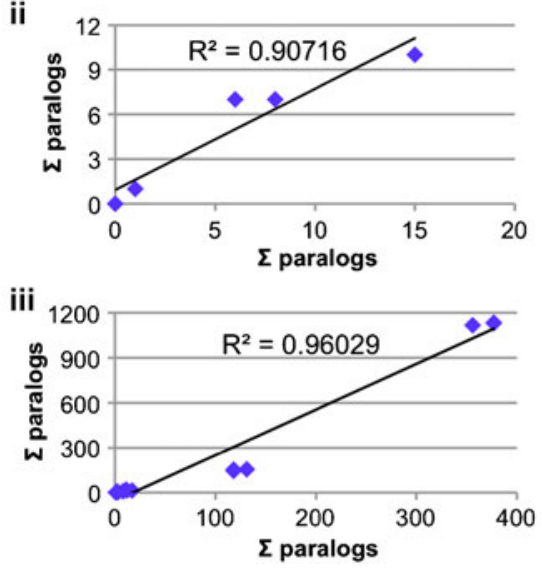

D

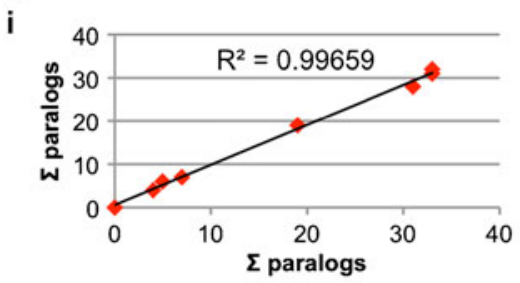

ii

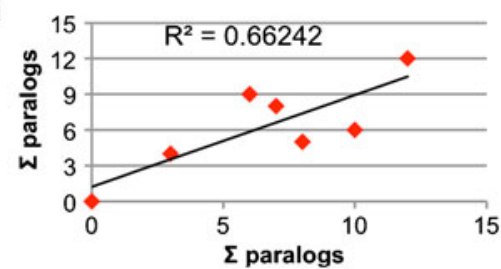

iii

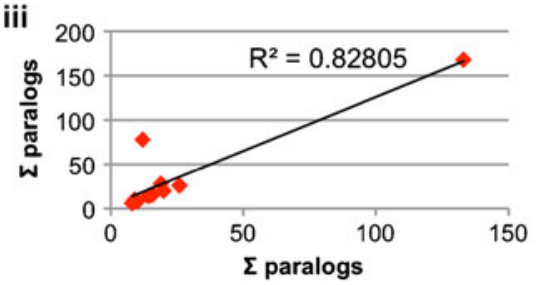

B

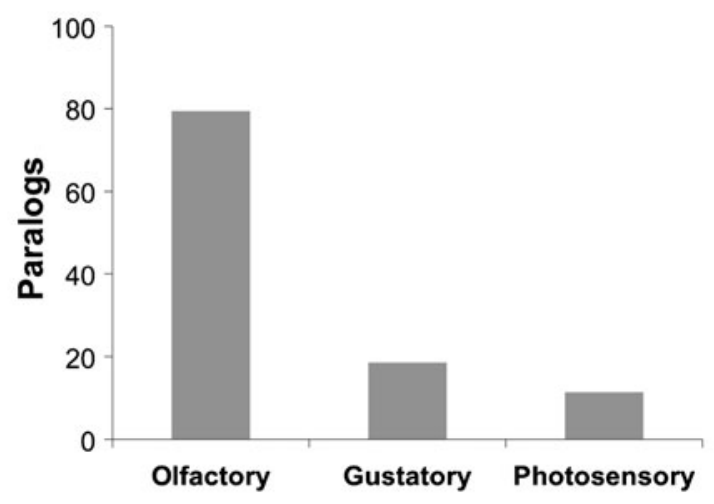

E

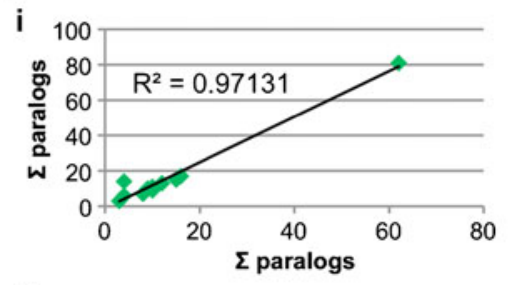

ii

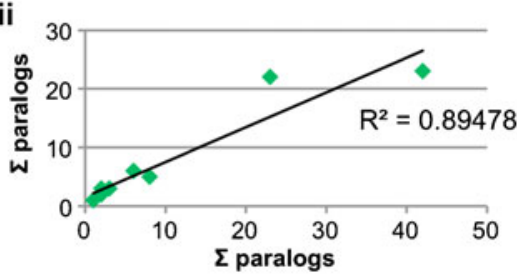

iii

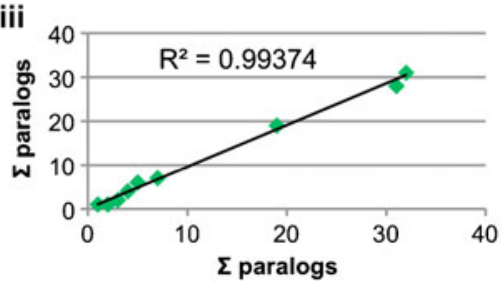

Figure 2. Gene duplication forms a source of novelty for all sensory modalities, in particular the olfactory circuit. (A) Box plots representing the total paralogue count (log scale) for each signalling pathway partitioned by sensory modality and species. (B) Average number of paralogues for each sensory modality across all phyla organized by sensory modality. (C-E) Graphs plotting correlation of the concordance between the number of paralogues within each phylum (i, nematode; ii, insect; iii, mammal) for the olfactory (C, i-iii), gustatory (D, i-iii), and photoexcitation (E, i-iii) pathways.

\section{Gene duplication and sensory systems: a heterogeneous strategy?}

The olfactory system is charged with decoding an incredibly diverse array of distinct stimuli. Olfactory receptor neurons converge onto the olfactory bulb in mammals, and the antennal lobe in Drosophila (Buck 2000; Hallem et al. 2006; Voshall and Stocker 2007). In each case, multiple olfactory neurons project onto overlapping glomeruli, which help decrease noise and heightens sensitivity. In C. elegans there are multiple olfactory receptors expressed per primary sensory neuron (Troemel et al. 1995). Within the olfactory system, new receptors can generate entirely new sensory input, and new regulators can create novelty by modulat- ing tuning, increasing discriminatory power or accelerating processing speed. Therefore, in the olfactory systems of nematodes, insects and mammals, gene duplication events may serve as the ideal mode of flexibility to maximize predictions about the environment while only incrementally modulating energy demands. Unlike the olfactory system, gene duplications of signalling machinery, and thus architecture, of the visual system in mammals and insects would likely require great energy demands but perhaps only achieve more resolving power. In $C$. elegans the photosensory circuit facilitates a basic nonocular phototaxis response that references an animal's orientation within the soil by detecting short wavelength light, and so genetic accretion may have 
a limited benefit within the photosensory circuit of $C$. elegans (Edwards et al. 2008; Liu et al. 2010). The gustatory system may represent a hybrid state as the benefits of gene duplication may be similar to those in the olfactory system in that new gustatory input could be generated through novelty. However, unlike the olfactory system, the gustatory architecture is more complex in insects and mammals, and so the energy costs associated with more novelty may outweigh the benefits. In Drosophila, the gustatory organs are distributed over the entire body (Voshall and Stocker 2007). Unlike the antennal lobe of the olfactory system of Drosophila, the gustatory neurons all over the body target the suboesophageal ganglion (SOG), and unlike the antennal lobe, the SOG does not exhibit any structural divisions such as the glomeruli of the olfactory system (Voshall and Stocker 2007). Further, in mammals the gustatory receptors are partitioned into specialized cells that decode specific tastes (sweet, sour, salty, bitter and umami), and each receptor employs a different form of signal transduction (Chandrashekar et al. 2006; Huang et al. 2006). Therefore, the benefits of genetic accretion at the receptor or even regulator level may only benefit a specific subclass of gustatory receptor neurons, and this compartmentalization perhaps dilutes, relatively at least, the selective pressure driving novelty through gene expansion across the gustatory circuit.

\section{Conclusion}

The goal of our analysis was to conduct a pair wise examination of the molecular evolution of sensory signalling pathways within divergent phyla. From this analysis we have found that sensory signalling pathway members undergo unusually high levels of constraint which are in fact higher than the level of global constraint across the genome in each case. However, within each signalling pathway we also reveal a conserved theme of large gene duplication events which are conserved within each phylum. These findings lead us to suggest a 'sessile' mechanism of sensory pathway evolution, which on one side facilitates bursts of gene duplications and relaxed selection, and on the other side is anchored by unusually high levels of selective constraint that preserve core sensory functions. Our findings are not a complete picture of how sensory modalities can evolve, but it is our hope that as more molecular data emerges on sensory circuits within diverse systems, it will yield more detailed models and descriptions of the molecular evolution of sensory signalling pathways, and through this analysis we can ultimately build a robust evo-devo picture of how sensory systems evolve.

\section{Acknowledgements}

We would like to thank The George Washington University Columbian College of Arts and Sciences and Department of Biological Sciences for funding to D. O'Halloran and C. He. We thank our anonymous reviewers for their helpful feedback and suggestions.

\section{References}

Buck L. B. 2000 The molecular architecture of odor and pheromone sensing in mammals. Cell 100, 611-618.

Buck L. and Axel R. 1991 A novel multigene family may encode olfactory receptors: a molecular basis for odor recognition. Cell 65, 175-187.

Chalasani S. H., Chronis N., Tsunozaki M., Gray J. M., Ramot D., Goodman M. B. and Bargmann C. I. 2007 Dissecting a circuit for olfactory behaviour in Caenorhabditis elegans. Nature $\mathbf{4 5 0}$, 63-70.

Chandrashekar J., Hoon M. A., Ryba N. J. and Zuker C. S. 2006 The receptors and cells for mammalian taste. Nature 444, 288-294.

Edgar R. C. 2004 MUSCLE: a multiple sequence alignment method with reduced time and space complexity. BMC Bioinformatics $\mathbf{5}$, 113.

Edwards S. L., Charlie N. K., Milfort M. C., Brown B. S., Gravlin C. N., Knecht J. E. and Miller K. G. 2008 A novel molecular solution for ultraviolet light detection in Caenorhabditis elegans. PLoS Biol. 6, e198.

Eisthen H. L. 2002 Why are olfactory systems of different animals so similar? Brain Behav. Evol. 59, 273-293.

Gaunt M. W. and Miles M. A. 2002 An insect molecular clock dates the origin of the insects and accords with palaeontological and biogeographic landmarks. Mol. Biol. Evol. 19, 748-761.

Guindon S. and Gascuel O. 2003 A simple, fast, and accurate algorithm to estimate large phylogenies by maximum likelihood. Syst. Biol. 52, 696-704.

Hallem E. A., Dahanukar A. and Carlson J. R. 2006 Insect odor and taste receptors. Annu. Rev. Entomol. 51, 113-135.

Huang A. L., Chen X., Hoon M. A., Chandrashekar J., Guo W., Tränkner D. et al. 2006 The cells and logic for mammalian sour taste detection. Nature 442, 934-938.

Jacobs D. K., Nakanishi N., Yuan D., Camara A., Nichols S. A. and Hartenstein V. 2007 Evolution of sensory structures in basal metazoa. Integr. Comp. Biol. 47, 712-723.

Librado P. and Rozas J. 2009 DnaSP v5: a software for comprehensive analysis of DNA polymorphism data. Bioinformatics $\mathbf{2 5}$, 1451-1452.

Liu J., Ward A., Gao J., Dong Y., Nishio N., Inada H. et al. 2010 C. elegans phototransduction requires a $\mathrm{G}$ protein-dependent cGMP pathway and a taste receptor homolog. Nat. Neurosci. 13, 715722.

Nei M., Xu P. and Glazko G. 2001 Estimation of divergence times from multiprotein sequences for a few mammalian species and several distantly related organisms. Proc. Natl. Acad. Sci. USA 98, 2497-2502.

Sato K., Pellegrino M., Nakagawa, T., Nakagawa, T., Vosshall, L. B. and Touhara K. 2008 Insect olfactory receptors are heteromeric ligand-gated ion channels. Nature 452, 1002-1006.

Strausfeld N. J. and Hildebrand J. G. 1999 Olfactory systems: common design, uncommon origins? Curr. Opin. Neurobiol. 9, 634-639.

Stein L. D., Bao Z., Blasiar D., Blumenthal T., Brent M. R., Chen N. et al. 2003 The genome sequence of Caenorhabditis briggsae: a platform for comparative genomics. PLoS Biol. 2, e45.

Troemel E. R., Chou J. H., Dwyer N. D., Colbert H. A. and Bargmann C. I. 1995 Divergent seven transmembrane receptors are candidate chemosensory receptors in C. elegans. Cell 83, 207-218.

Voshall L. B. and Stocker R. F. 2007 Molecular architecture of smell and taste in Drosophila. Annu. Rev. Neurosci. 30, 505-533.

Wang H. Y., Chien H. C., Osada N., Hashimoto K., Sugano S., Gojobori T. et al. 2007 Rate of evolution in brain-expressed genes in humans and other primates. PLoS Biol. 5, e13. 
Wicher D., Schäfer R., Bauernfeind R., Stensmyr M. C., Heller R., Heinemann S. H. and Hansson B. S. 2008 Drosophila odorant receptors are both ligand-gated and cyclic-nucleotide-activated cation channels. Nature 452, 1007-1011.

Yang Z. 1997 PAML: a program package for the phylogenetic analysis by maximum likelihood. Computer applications in the biosciences. Cabios 13, 555-556.
Yang Z. and Nielsen R. 2000 Estimating synonymous and nonsynonymous substitution rates under realistic evolutionary models. Mol. Biol. Evol. 17, 32-43.

Young J. M., Friedman C., Williams E. M., Ross J. A., TonnesPriddy L. and Trask B. J. 2002 Different evolutionary processes shaped the mouse and human olfactory receptor gene families. Hum. Mol. Genet. 11, 535-546.

Received 12 February 2013, in final revised form 30 April 2013; accepted 2 May 2013

Published on the Web: 5 August 2013 